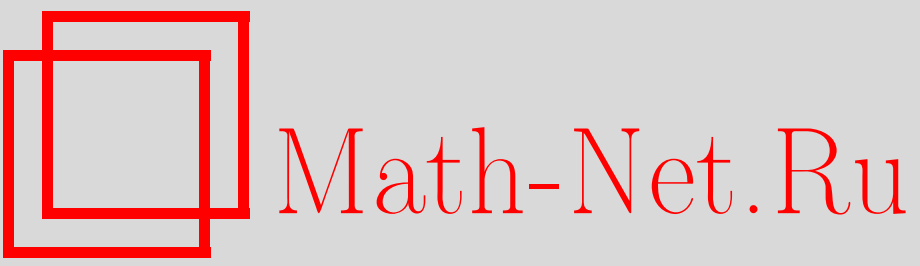

В. И. Денисов, М. И. Денисов, Н. В. Кравцов, В. Б. Пинчук, О проверке универсальности гравитационного взаимодействия оптическим методом, ТМФ, 1998, том 117, номер 3, 498-503

DOI: https://doi.org/10.4213/tmf948

Использование Общероссийского математического портала Math-Net.Ru подразумевает, что вы прочитали и согласны с пользовательским соглашением

http://www.mathnet.ru/rus/agreement

Параметры загрузки:

IP : 54.205 .225 .156

26 апреля 2023 г., 14:27:35 
ТЕОРЕТИЧЕСКАЯ

И МАТЕМАТИЧЕСКАЯ

ФИЗИКА

Том 117, № 3

декабрь, 1998

(C) 1998 г.

В. И. Денисов ${ }^{*}$, М. И. Денисов ${ }^{\dagger}$, Н.В. Кравцов* , В.Б. Пинчук*

\section{О ПРОВЕРКЕ УНИВЕРСАЛЬНОСТИ ГРАВИТАЦИОННОГО ВЗАИМОДЕЙСТВИЯ ОПТИЧЕСКИМ МЕТОДОМ}

Показано, что эксперименты с лазерным гироскопом позволяют проверить принцип универсальности гравитационного взаимодействия для фотонов по компоненте $g_{0 \varphi} \mathrm{c}$ точностью не хуже $10^{-16}$.

Проверка фундаментальных принципов физики, как известно, составляет одну из основных задач современного эксперимента. В области гравитации такими принципами являются принцип метричности гравитационного взаимодействия и принцип универсальности этого взаимодействия, получивший в научной литературе название принципа эквивалентности. Согласно этим принципам гравитационное воздействие осуществляется через метрический тензор псевдориманова пространства-времени $g_{i k}$ и этот тензор является единым для всех видов материи.

К настоящему времени проведен ряд экспериментов, проверяющих те или иные аспекты этих принципов со все возрастающей точностью. В частности, последние измерения отношения инертной и пассивной гравитационной масс тел лабораторных размеров подтвердили [1] справедливость этого принципа с относительной точностью $10^{-12}$.

Однако наибольший интерес представляют не опыты с телами макроскопических размеров, содержашими большое число различных частиц и полей (электроны, протоны, нейтроны, электромагнитное поле и т.п.), а эксперименты с частицами или полями одной природы. В этом случае возможное нарушение принципа универсальности для них будет проявляться в чистом виде и этот эффект не будет усредняться из-за присутствия частиц другой природы в изучаемой системе, для которых гравитационное воздействие, вообше говоря, может осушествляться через другой метрический тензор.

Одними из наиболее доступных экспериментов такого типа являются эксперименты с фотонами в гравитационном поле Солнечной системы. К настояшему времени уже

\footnotetext{
* Московский государственный университет, Москва, Россия

${ }^{\dagger}$ Научно-исследовательский институт источников тока, Краснодар, Россия
} 
проведено несколько экспериментов, в которых изучались гравитационные свойства фотонов. Это наблюдение эффектов гравитационного смешения частоты электромагнитной волны, искривления лучей и запаздывания электромагнитных сигналов при их распространении в гравитационном поле. Из этих экспериментов однозначно следует, что электромагнитные волны распространяются по геодезическим риманова пространства-времени и метрический тензор этого пространства с точностью порядка $10^{-10}$ совпадает с метрическим тензором для тел макроскопических размеров.

Дальнейшее усовершенствование измерительной техники и методики проведения этих экспериментов, к сожалению, не способно увеличить точность проверки принципа универсальности выше $10^{-12}-10^{-13}$. Поэтому возникает задача разработки принципиально новых экспериментов, которые позволили бы при современном уровне развития экспериментальной техники сушественно повысить точность проверки этого принципа. Одним из таких экспериментов, на наш взгляд, является эксперимент, используюший специфические свойства лазерных гироскопов.

Рассмотрим принципиальные возможности земного эксперимента по проверке принципа универсальности гравитационного взаимодействия с использованием фотонов, движушихся в лазерном гироскопе.

Как известно, для дифференциальных уравнений любого физического поля

$$
\widehat{L} \Psi=0
$$

можно построить [2] уравнение характеристик. В наиболее обшем случае оно принимает вид

$$
g^{i k} \frac{\partial S}{\partial x^{i}} \frac{\partial S}{\partial x^{k}}=m_{0}^{2} c^{2}
$$

где $g^{i k}$ - метрический тензор некоторого риманова пространства-времени, $m_{0}$ - масса покоя кванта рассматриваемого поля.

Метрический тензор риманова пространства-времени в этом уравнении возникает как следствие наличия нелинейностей при старших производных в уравнении поля, а также воздействия гравитационного поля. Таким образом, метрический тензор $g^{i k}$ целиком предопределяется выбором лагранжиана, а точнее, наличием тех или иных множителей при старших производных в нем.

В частности, в любой нелинейной теории, сформулированной, например, с использованием метрического тензора плоского пространства-времени и использующей ковариантные производные только по этой метрике, но содержащей нелинейности функций поля при старшей производной, фронт волны этого поля будет распространяться по геодезическим риманова пространства-времени, метрический тензор которого зависит от функций поля и метрического тензора плоского пространства-времени.

Одним из примеров такой теории является нелинейная электродинамика Борна-Инфельда [3], которая сформулирована в терминах метрического тензора плоского пространства-времени, а характеристики которой, как показано в работе [4], распространяются по геодезическим некоторого, вообше говоря, риманова пространства-времени. 
Вопрос о выборе метрического тензора - это вопрос о выборе коэффициентов при старших производных в лагранжиане поля. Согласно современным представлениям этот тензор должен быть единым для всех полей материи. Однако не исключается и высказанная еще Лобачевским идея, что различные физические поля могут описываться в терминах различных геометрий. В этом случае уравнение фронта для каждого физического поля по форме будет совпадать с уравнением (1), но содержать свой характерный для данного взаимодействия метрический тензор риманова пространства-времени. Очевидно, что до какого-то уровня эти метрические тензоры будут совпадать, а начиная с некоторого уровня их различие можно заметить на эксперименте.

Для проверки принципа универсальности в земных условиях с использованием фотонов предположим, что метрический тензор риманова пространства для фотонов $g_{i k}^{\mathrm{Ph}}$ гравитационном поле Солнечной системы слегка отличается от метрического тензора других полей материи, например нуклонов $g_{i k}^{\mathrm{N}}$. Так как основную часть массы Земли, Солнца и других тел Солнечной системы составляют нуклоны, то все тела будут двигаться, испытывая главным образом влияние метрического тензора $g_{i k}^{\mathrm{N}}$. Следовательно, проводя эксперименты с фотонами, при определенных условиях можно попытаться измерить разность $g_{i k}^{\mathrm{Ph}}-g_{i k}^{\mathrm{N}}$ или некоторые компоненты этой разности и проверить тем самым принцип универсальности с большей, чем ранее, точностью.

Рассмотрим систему отсчета, связанную с центром масс Земли. В гравитационном поле Солнца эту систему отсчета с достаточно хорошей точностью можно считать локально-инерциальной системой отсчета для нуклонов. Поэтому в некоторой окрестности центра масс Земли все частные производные первого порядка от метрического тензора $g_{i k}^{\mathrm{N}}$ будут пренебрежимо малы и сам метрический тензор в этой окрестности практически будет совпадать с галилеевым метрическим тензором: $g_{i k}^{\mathrm{N}}=\eta_{i k}$.

Предположим, что в этой системе отсчета находится лазерный гироскоп. Так как метрический тензор для электромагнитного поля $g_{i k}^{\mathrm{Ph}}$ может совпадать с метрическим тензором $g_{i k}^{\mathrm{N}}$, то локально-инерциальная система отсчета нуклонов будет неинерциальной для фотонов и ее вращение можно будет измерить по показаниям лазерного гироскопа.

Запишем метрический тензор $g_{i k}^{\mathrm{Ph}}$ в виде [5]

$$
g_{i k}^{\mathrm{Ph}}=g_{i k}^{\mathrm{N}}+h_{i k}
$$

где $h_{i k}$ - тензор, отличие которого от нуля означает нарушение принципа универсальности гравитационного взаимодействия.

В системе отсчета, связанной с центром масс Земли, это соотношение примет вид $g_{i k}^{\mathrm{Ph}}=\eta_{i k}+h_{i k}$. Изучим законы распространения плоской электромагнитной волны в этой метрике.

Так как волновой 4-вектор для электромагнитной волны удовлетворяет соотношению

$$
g_{m l}^{\mathrm{Ph}} k^{m} k^{l}=0
$$


то отсюда несложно найти закон дисперсии - зависимость частоты $\omega$ от трехмерных компонент $k^{\alpha}$ волнового вектора:

$$
\omega(\vec{k})=-\frac{c\left[h_{0 \alpha} k^{\alpha} \pm \sqrt{\left(h_{0 \alpha} k^{\alpha}\right)^{2}-g_{00}^{\mathrm{Ph}} g_{\alpha \beta}^{\mathrm{Ph}} k^{\alpha} k^{\beta}}\right]}{g_{00}^{\mathrm{Ph}}} .
$$

Учитывая, что $g_{i k}^{\mathrm{N}}=\eta_{i k}$, и ограничиваясь членами, линейными по компонентам малого тензора $h_{m l}$, будем иметь

$$
\omega(\vec{k})= \pm\left[1-\frac{h_{00}}{2}-\frac{h_{\alpha \beta} k^{\alpha} k^{\beta}}{2 \vec{k}^{2}}\right]-c h_{0 \alpha} k^{\alpha} .
$$

Изучим теперь, как будет влиять это дисперсионное уравнение на работу лазерного гироскопа. Лазерный гироскоп, как известно, представляет собой кольцевой высокодобротный оптический резонатор, внутри которого находится активная среда, обеспечивающая высокостабильную стационарную генерацию с длиной волны 0.63 мкм двух встречных волн, разность частот которых определяется невзаимностью оптического резонатора, т.е. нарушением идентичности условий распространения встречных волн (см. например, [6]).

Именно такая неидентичность и будет иметь место в случае нарушения принципа эквивалентности (неравенство нулю компоненты $h_{0 \alpha}$ ).

Расчет относительной разности частот $\Delta \omega / \omega$ встречных волн лазерного гироскопа проводится совершенно аналогично расчету в работе [7]. Относительная разность частот генерации двух электромагнитных волн, распространяюшихся в лазерном гироскопе во взаимно противоположных направлениях, оказывается равной удвоенной величине компоненты $h_{0 \varphi}$ :

$$
\frac{\Delta \omega}{\omega}=\frac{\omega(\vec{k})-\omega(-\vec{k})}{\omega}=-2 h_{0 \varphi}
$$

Поскольку серийные лазерные гироскопы с линейным размером порядка 10 см в соединении со специализированным микрокомпьютером позволяют регистрировать отношение $\Delta \omega / \omega$ с точностью на уровне $10^{-16}$, то из выражения (2) непосредственно следует, что, измеряя $\Delta \omega / \omega$ при разных ориентациях лазерного гироскопа относительно $\vec{\Omega}$ - вектора угловой скорости движения Земли по орбите вокруг Солнца, можно измерить компоненту $h_{0 \varphi}$, характеризующую нарушение принципа универсальности, с точностью не хуже чем $10^{-16}$

Отметим, что современный уровень лазерного приборостроения позволяет реализовать лазерные гироскопы, обладаюшие точностью измерения угловых скоростей порядка 0.001 углового градуса в час [6], что соответствует $\delta \omega / \omega \leqslant 10^{-17}$.

При расчете эффекта мы предполагали, что лазерный гироскоп находится в покое в системе отсчета, которая в гравитационном поле Солнца является локально-инерциальной для нуклонов. Однако лабораторная система отсчета, находящаяся на Земле и доступная для проведения эксперимента, не является локально-инерциальной из-за участия во вращательном движении вокруг оси Земли и наличия земного гравитационного 
поля. Основным препятствием для осушествления данного эксперимента является собственное врашение Земли с частотой $\Omega_{\oplus} \simeq 2 \pi /$ сутки $\simeq 0.7 \cdot 10^{-4}$ рад/с, которое вызывает появление большого маскируюшего эффекта в показаниях лазерного гироскопа и затрудняет получение высокой точности.

Устранить этот маскируюший эффект в принципе можно двумя путями: либо вычесть влияние вращения Земли из результирующих показаний лазерного гироскопа при обработке результатов экспериментов, либо поместить лазерный гироскоп в такие условия, при которых он не будет испытывать влияния вращения Земли.

Легко понять, что первый путь является менее привлекательным. Действительно, при чувствительности $\Delta \omega / \omega \simeq 10^{-16}$ лазерный гироскоп способен зарегистрировать врашения на уровне $\delta \Omega \simeq 10^{-7}$ рад/с, поэтому для достижения предельной точности проверки принципа эквивалентности необходимо знать вариации угловой частоты вращения Земли с точностью $\delta \Omega_{\oplus} \simeq 10^{-7}$ рад/с $\left(\delta \Omega_{\oplus} / \Omega_{\oplus} \simeq 10^{-3}\right)$, что довольно трудно осушествить на практике.

Более перспективным, на наш взгляд, является второй путь: поместить лазерный гироскоп на трехосную механическую гироскопическую платформу и тем самым устранить влияние врашения Земли на показания лазерного гироскопа аппаратными средствами.

В этом случае, если не учитывать влияния собственного гравитационного поля Земли $\left(U_{\oplus} \simeq 0.7 \cdot 10^{-9}\right)$, система отсчета, связанная с лазерным гироскопом, будет свободно падающей в гравитационном поле Солнца (т.е. локально-инерциальной системой отсчета) для нуклонов, составляющих основную массу Земли и механической гироскопической платформы. Поэтому в области лазерного гироскопа с большой точностью будет выполняться условие $g_{i k}^{\mathrm{N}}=\eta_{i k}$, и он будет измерять компоненту $h_{0 \varphi}$ в "чистом" виде.

Если учесть существование собственного поля Земли, то в линейном по $U_{\oplus} \sim 10^{-9}$ приближении в системе отсчета, связанной с лазерным гироскопом, будем иметь

$$
\begin{aligned}
g_{00}^{\mathrm{N}} & =1-2 U_{\oplus}, \quad g_{\alpha \beta}^{\mathrm{N}}=-\delta_{\alpha \beta}\left[1+2 U_{\oplus}\right], \\
g_{00}^{\mathrm{Ph}} & =1-2 U_{\oplus}+h_{00}, \quad g_{0 \alpha}^{\mathrm{Ph}}=h_{0 \alpha}, \\
g_{\alpha \beta}^{\mathrm{Ph}} & =-\delta_{\alpha \beta}\left[1+2 U_{\oplus}\right]+h_{\alpha \beta} .
\end{aligned}
$$

Так как на работу лазерного гироскопа основное влияние оказывают компоненты $g_{0 \varphi}^{\mathrm{Ph}}$ метрического тензора, то фактически в лабораторном эксперименте с лазерным гироскопом, помешенным на механическую гироскопическую платформу, будет измеряться величина $h_{0 \varphi}$ с точностью $\sim 10^{-16}$ независимо от влияния $U_{\oplus}$.

В заключение отметим, что для получения указанной вьше точности измерения компоненты $h_{0 \varphi} \sim 10^{-16}$ необходимо, чтобы механические гироскопы платформы обеспечивали стабилизацию лазерного гироскопа от врашения Земли на уровне $\delta \omega \approx 10^{-3} \Omega_{\oplus}$, где $\Omega_{\oplus}$ - угловая скорость вращения Земли.

Современньй уровень развития гироскопической техники, как известно, позволяет осуществить такую стабилизацию.

Настоящая работа выполнена при частичной поддержке РФФИ, грант.№ 98-02-17448а. 


\section{Список литературы}

[1] В. Б. Брагинский, В. И. Панов. ЖЭТФ. 1971. Т. 61. № 3. С. 873.

[2] I. P. Denisova, M. Dalal. J. Math. Phys. 1997. V. 38. № 11. P. 5820.

[3] M. Born, L. Infeld. Proc. Roy. Soc. 1934. V. A144. P. 425.

[4] Д. И. Блохинцев. Пространство и время в микромире. М.: Наука, 1970. С. 43.

[5] I. P. Denisova, B. V. Mehta. General Relativ. Gravit. 1997. V. 29. № 5. P. 583.

[6] J. R. Wilkinson. Progress in Quantum Electronics. 1987. V. 11. P. 1.

[7] В. И. Денисов, М. И. Денисов. Проверка принципа эквивалентности Эйнштейна с помощью лазерного гироскопа в космосе. Препринт НИИЯФ МГУ № 95-25/389. М.: НИИЯФ, 1995.

Поступила в редакцию 20.IV.1998 г.

\section{Исправление к статье:}

Ю. А. Кашлев, Н.М. Садыков "Неравновесная статистическая термодинамика каналированных частищ. Переход к частицам, движушимся с тепловыми скоростями" (ТМФ. 1998. Т. 116. № 3. С. 442-455)

Формула (25) должна выглядеть следуюшим образом:

$$
\Phi=\left.\left(\frac{m}{m_{\mathrm{e}}}\right)\left\{E_{0} \psi_{\mathrm{cr}}^{2} R /\left(-\frac{d E}{d t}\right)_{\mathrm{e}}\right\}\right|_{E_{0}=\mathrm{const}} .
$$

Редакция приносит авторам свои извинения. 